\title{
Two Phase 3 Trials of Dupilumab versus Placebo in Atopic Dermatitis
}

TO THE EDITOR: The conclusions of Simpson and colleagues (Dec. 15 issue) ${ }^{1}$ regarding the effectiveness and quality-of-life-enhancing capacity of dupilumab in patients with atopic dermatitis open new perspectives in the strategies of treatment of allergic diseases, because interleukin-4 and interleukin-13 are "type 2 inflammatory cytokines that may be important drivers of atopic or allergic diseases." Given the good results in patients with asthma ${ }^{2}$ or nasal polyposis, ${ }^{3}$ we as pediatricians look with interest to the possibilities arising from these studies involving adults. However, the higher rate of allergic conjunctivitis in the dupilumab-treated population than in the placebo group in this trial runs against these wishes. Although we agree with the authors that "further studies on the causes of conjunctivitis are warranted," could these be cases of atopic keratoconjunctivitis rather than simple allergic conjunctivitis? This condition is often linked to atopic dermatitis. ${ }^{4}$ If so, could it be hypothesized that the blockage of interleukin- 4 and interleukin-13 increases the activity of the specific ligands involved in atopic keratoconjunctivitis (e.g., the OX40 ligand) in the eye, an immunologically privileged area? $?^{5}$ This would limit the potential uses of dupilumab in the allergy field.

Maurizio Mennini, M.D.

Lamia Dahdah, M.D.

Alessandro Fiocchi, M.D.

Bambino Gesù Children's Research Hospital

Rome, Italy

maurizio.mennini@gmail.com

No potential conflict of interest relevant to this letter was reported.

1. Simpson EL, Bieber T, Guttman-Yassky E, et al. Two phase 3 trials of dupilumab versus placebo in atopic dermatitis. $\mathrm{N}$ Engl J Med 2016;375:2335-48.

2. Sy H, Bielory L. Atopic keratoconjunctivitis. Allergy Asthma Proc 2013;34:33-41.

3. Bachert C, Mannent L, Naclerio RM, et al. Effect of subcutaneous dupilumab on nasal polyp burden in patients with chronic sinusitis and nasal polyposis: a randomized clinical trial. JAMA 2016;315:469-79.

4. Wenzel S, Ford L, Pearlman D, et al. Dupilumab in persistent asthma with elevated eosinophil levels. N Engl J Med 2013;368: 2455-66.

5. Masli S, Vega JL. Ocular immune privilege sites. Methods Mol Biol 2011;677:449-58.

DOI: $10.1056 /$ NEJMc1700366
THE AUTHORS REPLY: In trials of dupilumab involving patients with atopic dermatitis, conjunctivitis occurred more often in patients who had severe atopic dermatitis or coexisting allergic conjunctivitis ( $>25 \%$ of the enrolled patients). More than $90 \%$ of the adverse events of conjunctivitis were mild or moderate in severity, and more than $75 \%$ resolved or were resolving during study treatment. In the SOLO trials, 1 of 920 patients who were treated with dupilumab discontinued study treatment because of conjunctivitis. Dupilumab was not associated with a higher rate of adverse events of conjunctivitis than placebo in trials involving patients with asthma or nasal polyposis, ${ }^{1,2}$ which suggests an atopic dermatitis-specific mechanism, not an inherent effect of dupilumab.

The cause is unclear. Not all cases were diagnosed by ophthalmologists. Findings did not typically suggest atopic keratoconjunctivitis. Two clinical phenotypes were noted: blepharoconjunctivitis that was consistent with atopic dermatitis in the eyelids and periorbital area, and conjunctivitis with limbal accentuation. The incidence of atopic keratoconjunctivitis was less than $0.5 \%$ across all groups and was not higher in the dupilumab group than in the placebo group. Although the cornea is immune-privileged, the rest of the ocular surface, eyelids, and periorbital skin are not.

There is an inverse relationship between serum concentrations of dupilumab and conjunctivitis, which suggests that local undertreatment, possibly due to higher target burden or lower tissue distribution, may play a role. In our trials and others, ${ }^{1,2}$ dupilumab effectively inhibited the inflammation of type 2 helper T (Th2) cells in patients with atopic dermatitis, asthma, or nasal polyposis. Similar Th2-cell inflammatory pathways are involved in atopic keratoconjunctivitis.

Eric L. Simpson, M.D.

Oregon Health and Science University

Portland, OR

simpsone@ohsu.edu

Bolanle Akinlade, M.D.

Marius Ardeleanu, M.D.

Regeneron Pharmaceuticals

Tarrytown, NY

Since publication of their article, the authors report no further potential conflict of interest. 
1. Wenzel S, Castro M, Corren J, et al. Dupilumab efficacy and safety in adults with uncontrolled persistent asthma despite use of medium-to-high-dose inhaled corticosteroids plus a long-acting beta-agonist: a pivotal dose-ranging study. Lancet 2016;388:31-44.
2. Bachert C, Mannent L, Naclerio RM, et al. Effect of subcutaneous dupilumab on nasal polyp burden in patients with chronic sinusitis and nasal polyposis: a randomized clinical trial. JAMA 2016;315:469-79.

DOI: 10.1056/NEJMc1700366

\section{Short-Term versus Long-Term Blood Storage}

TO THE EDITOR: In the Informing Fresh versus Old Red Cell Management (INFORM) study, Heddle et al. (Nov. 17 issue) ${ }^{1}$ suggest that the debate over red-cell storage and outcome after transfusion is now closed. According to the accompanying editorial, ${ }^{2}$ there may no longer be an issue regarding the freshness of red cells for transfusion. Although we admire the quality of all five recent, large cited studies on this topic ${ }^{2}$ (which have covered such diverse topics as the relationship between the age of blood and type of processing to patients' outcomes), we consider this conclusion to be questionable.

Previous studies have stressed the safety and survival benefit of a restrictive policy versus a liberal policy for red-cell transfusion, which suggests a downside to such transfusion. ${ }^{3}$ Red-cell storage lesions may explain this observation. Stored red cells have both reversible and irreversible lesions; the latter occur from the second week onward. ${ }^{4}$ However, recent studies have compared red cells that were stored for 7 to 10 days with those that were stored for 15 to 20 days in other words, comparing old red cells with older red cells. Other studies have suggested that the transfusion of blood from donors who have characteristics associated with storage problems (so-called bad donors) ${ }^{5}$ has resulted in poor outcomes among the recipients, an effect that was not analyzed in the current trials. We thus encourage the continuation of trials of really fresh versus stored red cells and the identification of donor characteristics associated with good recipient outcomes.

Olivier Garraud, M.D., Ph.D.

Institut National de la Transfusion Sanguine

Paris, France

ogarraud@ints.fr

Jean-Daniel Tissot, M.D., Ph.D.

Transfusion Interrégionale Croix Rouge Suisse Epalinges, Switzerland
Alexander P. Vlaar, M.D., Ph.D.

Academic Medical Center

Amsterdam, the Netherlands

No potential conflict of interest relevant to this letter was reported.

1. Heddle NM, Cook RJ, Arnold DM, et al. Effect of short-term vs. long-term blood storage on mortality after transfusion. N Engl J Med 2016;375:1937-45.

2. Tobian AA, Ness PM. Red cells - aging gracefully in the blood bank. N Engl J Med 2016;375:1995-7.

3. Holst LB, Petersen MW, Haase N, Perner A, Wetterslev J. Restrictive versus liberal transfusion strategy for red blood cell transfusion: systematic review of randomised trials with metaanalysis and trial sequential analysis. BMJ 2015;350:h1354.

4. Prudent M, Tissot JD, Lion N. In vitro assays and clinical trials in red blood cell aging: lost in translation. Transfus Apher Sci 2015;52:270-6.

5. Chassé M, McIntyre L, English SW, et al. Effect of blood donor characteristics on transfusion outcomes: a systematic review and meta-analysis. Transfus Med Rev 2016;30:69-80.

DOI: $10.1056 / N E J M c 1700464$

TO THE EDITOR: The study by Heddle and colleagues is limited in what it actually addresses by design, since it specifically shows only that the length of red-cell storage is immaterial to mortality in the context of the current practice of red-cell transfusion aggregated across diverse clinical settings. Given that design, the study does not address whether any of the transfusions were essential, since most of the patients at highest risk were excluded. Therefore, it is uncertain whether a between-group difference could have been detected. Without knowing whether the study design could have detected a true difference, it remains possible that an important association between storage length and outcomes exists.

There remains much controversy as to when and in which clinical settings transfusions are clearly beneficial to mortality, ${ }^{1,2}$ with wide variability in transfusion practice ${ }^{3}$ and, arguably, still much overuse. This study leaves unaddressed important questions - notably, whether storage 